\title{
The Complex $\alpha-\mu$ Fading Channel with OFDM Application
}

\author{
Wander H. M. Freitas, ${ }^{1}$ Roberto C. D. V. Bomfin, ${ }^{2}$ \\ Rausley A. A. de Souza, ${ }^{1}$ and Michel Daoud Yacoub ${ }^{3}$ \\ ${ }^{1}$ National Institute of Telecommunications (Inatel), P.O. Box 05, 37540-000 Santa Rita do Sapucaí, MG, Brazil \\ ${ }^{2}$ Vodafone Chair Mobile Communication Systems, Technische Universität Dresden, 01062 Dresden, Germany \\ ${ }^{3}$ Wireless Technology Laboratory (WissTek), Department of Communications, School of Electrical and Computation Engineering, \\ State University of Campinas, Campinas, SP, Brazil
}

Correspondence should be addressed to Rausley A. A. de Souza; rausleyaas@gmail.com

Received 9 December 2016; Accepted 4 July 2017; Published 17 August 2017

Academic Editor: Giuseppe Castaldi

Copyright (C) 2017 Wander H. M. Freitas et al. This is an open access article distributed under the Creative Commons Attribution License, which permits unrestricted use, distribution, and reproduction in any medium, provided the original work is properly cited.

The aims of this paper are threefold: (i) to present a model for the complex $\alpha-\mu$ fading channel; (ii) to propose an efficient, simple, and general method to generate complex $\alpha$ - $\mu$ samples; (iii) to make use of this channel in order to assess the bit error rate performance of an OFDM system. An analytical framework is then used, whose output is validated through Monte Carlo simulation. Several important conclusions concerning the system performance as a function of the channel parameters, namely, nonlinearity, clustering, and power imbalance of in-phase and quadrature components, are drawn.

Dedicated to the memory of our beloved and brilliant friend Wander Henrique Machado Freitas, the first author of this paper, who passed away in December 16, 2016, one week after the submission of this paper

\section{Introduction}

Wireless communication channels are subject to fading, with the received signal varying in a random manner $[1,2]$. Because signal variation deteriorates the performance of the communication systems, the correct characterization of the phenomenon is of paramount importance. It is widely understood that the signal oscillates on a statistical basis with several short-term distributions adequately describing this variation. One of such distributions, arising from the $\alpha-\mu$ fading model [3], describes the small-scale variations of the signal in a highly diffuse scattering environment. It is a general, flexible, and mathematically easily tractable distribution which models the nonlinearity of the propagation medium, given by the parameter $\alpha$, as well as the multipath clustering of the radio waves, given by the parameter $\mu$. It includes important and widely accepted distributions such as Gamma, Nakagami- $m$, exponential, Weibull, one-sided Gaussian, and Rayleigh. Specifically, for $\alpha=2$ and $\mu=m$, the $\alpha-\mu$ model becomes the Nakagami- $m$ one with $m$ representing the degree of fading. For $\mu=1, \alpha-\mu$ deteriorates into Weibull. It is noteworthy that $\alpha-\mu$, as well as Nakagami- $m$, was initially conceived to model only the signal envelope. Therefore, their corresponding phases constitute an open matter. In several applications, however, the characterization of the phase may be critical, and a model for it is certainly of interest. In the absence of the knowledge of the phase statistics, and for simplicity, some researchers have adopted the uniform phase distribution. However, this is a rather simplistic assumption, since it is hard to imagine a fading signal with uniform distribution whose envelope approximately ranges from Hoyt to Rice, Hoyt and Rice themselves having nonuniform phases. In an attempt to fill this gap, in [4], a complex fading model leading to Nakagami- $m$ envelope and nonuniform phase distribution was proposed. Such a model was then improved in [5] to account for power, or, equivalently, clustering, imbalance between in-phase and quadrature components. It is noteworthy that the Nakagami- $m$ complex model has already been validated in practice through field measurements [6]. 
The increase of the demand for wireless services has led the systems to operate in a variety of environments. In particular, the diversity of scenarios envisaged for the fifth generation (5G) applications at the millimeter wave (mmWave) band will certainly lead to a variety of propagation conditions, which may not be characterized by simpler fading models. The widely used distributions, such as Rayleigh [7], Hoyt [8], Rice [9], Nakagami-m [10], and Weibull [11], will still find their place, of course, but they are less flexible for encompassing more sophisticated situations. One general and rather flexible, yet simple, model such as $\alpha-\mu$ [3] may serve this purpose. Interestingly enough, the $\alpha-\mu$ fading distribution has shown yielding an excellent curve fitting performance with real field data collected at a mmWave band, namely, $60 \mathrm{GHz}[12]$.

In [13], a very general, probably the most general, complex short-term model, the $\alpha-\eta-\kappa-\mu$ fading model, was proposed. Such a model comprises as special cases a number of important distributions, for example, $\alpha-\mu, \eta-\mu, \kappa-\mu$, and $\eta$ $\kappa$ (Beckmann), already widely explored in the literature. Hence, it is possible to specialize the $\alpha-\eta-\kappa-\mu$ model into the complex $\alpha$ - $\mu$ scenario with an aim at assessing some wireless performance metrics upon which in-phase and quadrature distributions may impact. And this is one of the objectives of this paper.

Currently, an enormous variety of waveforms are considered to be potential candidates for the $5 \mathrm{G}$ air interface. They include (i) Single-Carrier Frequency Division Multiplexing, also called differently cyclic prefix (CP) DFT-Spread-OFDM (already used in 4G LTE uplink), G-DFT-s-OFDM [14]; (ii) Zero-Tail (ZT) or Unique-Word (UW) DFT-Spread-OFDM $[15,16]$, UW-OFDM, GFDM [17], CP-OFDM (already used in the 4G LTE downlink); (iii) Resource-Block-Filtered OFDM, Filter-Bank-Multicarrier (FBMC), and Universal Filter Multicarrier (UFMC). As can be seen, the OFDM technique is omnipresent in the $5 \mathrm{G}$ waveform proposals. The OFDM technique shall certainly remain as the root framework for the new $5 \mathrm{G}$ waveform design, with some optimization to support the new $5 \mathrm{G}$ requirements $[18,19]$.

This paper makes use of the complex $\alpha-\mu$ fading channel to assess the bit error rate (BER) performance of a BPSKbased OFDM system. In particular, it generalizes the results of [20], in which such a performance metric was carried out for the Nakagami- $m$ case. The generalization approaches two important issues concerning their impact on the system performance: (i) the effect of the nonlinearity parameter; (ii) the effect of the power (or, equivalently, clustering) imbalance between in-phase and quadrature components. The analytical results are contrasted with simulation and a perfect agreement between them is found.

As a by-product of our main investigation, we propose an efficient method for generating complex $\alpha-\mu$ random variables, in which clustering imbalance is found. It is noteworthy that the proposed $\alpha-\mu$ variate generation method is simple and can be used in several other scenarios.

The paper is organized as follows. In Section 2, we present the complex $\alpha-\mu$ model highlighting its main statistics, namely, the probability density function (PDF) of the envelope, phase, and the joint PDF of in-phase and quadrature components. Section 3 presents an efficient, simple, and general method to generate $\alpha-\mu$ random variables. Section 4 details the procedure used to estimate the BER in a given subcarrier. The results both for the simulation algorithm and for the BER performance are shown in their respective sections. Finally, Section 5 concludes the paper.

\section{The Complex $\alpha-\mu$ Model}

This section introduces the complex $\alpha-\mu$ model. The complex $\alpha-\mu$ random variable $Z$ is defined as

$$
Z=X+j Y
$$

where $X$ and $Y$ correspond to the in-phase and quadrature components. Define $R=|Z|$ and $\Theta=\arg (Z)$ as the envelope (modulus) and the phase (argument) of the complex $\alpha-\mu$ variate, respectively. The $\operatorname{PDF} f_{R}(r)$ of the envelope $R$ is known to be given by [3]

$$
f_{R}(r)=\frac{\alpha \mu^{\mu} r^{\alpha \mu-1}}{\widehat{r}^{\alpha \mu} \Gamma(\mu)} \exp \left(-\mu \frac{r^{\alpha}}{\widehat{r}^{\alpha}}\right),
$$

with $r \geq 0$, and (i) $\alpha>0$ describes the nonlinearity of the propagation medium; (ii) $\mu>0$ represents the number of multipath clusters adding up at the receiver; (iii) $\widehat{r}=\sqrt[\alpha]{\mathbb{E}\left[R^{\alpha}\right]}$ is the $\alpha$-root mean value of the envelope; (iv) $\mathbb{E}[\cdot]$ is the expectation operator; (v) and $\Gamma(\cdot)$ is the Gamma function [21, eqn. (6.1.1)]. As already mentioned, as far as the envelope statistics are concerned, the $\alpha-\mu$ random variable includes the Gamma (and its discrete versions Erlang and central Chi-squared), Nakagami-m (and its discrete version Chi), exponential, Weibull, one-sided Gaussian, and Rayleigh ones. The Weibull distribution can be obtained from the $\alpha$ - $\mu$ distribution by setting $\mu=1$. From the Weibull distribution, by setting $\alpha=2$, the Rayleigh distribution results. Still from the Weibull distribution, the negative exponential distribution is obtained by setting $\alpha=1$. The Nakagami- $m$ distribution can be obtained from the $\alpha-\mu$ distribution by setting $\alpha=2$. From the Nakagami- $m$ distribution, by setting $\mu=1$, the Rayleigh distribution results. Still from the Nakagami- $m$ distribution, the one-sided Gaussian distribution is obtained by setting $\mu=1 / 2$.

Starting out from the $\alpha-\eta-\kappa-\mu$ fading [13] and specializing its parameters as $\kappa \rightarrow 0$ and $\eta=1$, it is possible to arrive at the corresponding $\alpha$ - $\mu$ phase-envelope joint PDF. From there, the in-phase-quadrature joint PDF can be found. On the other hand, as detailed in [13], the nonlinearity parameter does not affect the phase statistics, so that its phase $\operatorname{PDF} f_{\Theta}(\theta)$ is that of Nakagami- $m$. Hence

$$
\begin{aligned}
& f_{\Theta}(\theta) \\
& =\frac{\Gamma(\mu)}{2^{\mu} \Gamma(((1+p) / 2) \mu) \Gamma(((1-p) / 2) \mu)} \frac{|\sin (2 \theta)|^{\mu-1}}{|\tan (\theta)|^{p \mu}},
\end{aligned}
$$

with $-\pi \leq \theta \leq \pi$ and $-1 \leq p \leq 1$ is a power (or clustering) imbalance parameter. This parameter is intrinsically connected to the multipath clustering effect as [5] (the definition 
of the clustering imbalance parameter in this paper keeps that used in [5] and is different from that used in [13]; of course, one is related to the other as detailed in [13]). From [3], we know that the $k$ th moment of the envelope-based $\alpha-\mu$ random variable is given by $\mathbb{E}\left[R^{k}\right]=\widehat{r}^{k} \Gamma(\mu+k / \alpha) /\left(\mu^{k / \alpha} \Gamma(\mu)\right)$, which makes us able to set

$$
\widehat{r}=\sqrt{\frac{\mu^{2 / \alpha} \Gamma(\mu) \mathbb{E}\left[R^{2}\right]}{\Gamma(\mu+2 / \alpha)} .}
$$

Again, particularizing $\alpha-\eta-\kappa-\mu$ fading model as required, the $\alpha-\mu$ joint $\operatorname{PDF} f_{R, \Theta}(r, \theta)$ is then found as

$$
\begin{aligned}
& f_{R, \Theta}(r, \theta) \\
& =\frac{\alpha \mu^{\mu} r^{\alpha \mu-1} \exp \left(-\mu\left(r^{\alpha} / \widehat{r}^{\alpha}\right)\right)|\sin (2 \theta)|^{\mu-1}}{2^{\mu \widehat{r}^{\alpha \mu}} \Gamma(((1+p) / 2) \mu) \Gamma(((1-p) / 2) \mu)|\tan (\theta)|^{p \mu}} .
\end{aligned}
$$

It can be seen from (5) that envelope and phase are independent variates. This is coherent with the complex Nakagami$m$ model $[4,5]$ in which the independence condition arose naturally out of the derivation of the model. As a particular case, the following are noted: (i) $p=0$ denotes the power balance condition; (ii) $p=1$ implies that the all signal power is concentrated in the in-phase component; (iii) $p=-1$ implies that all the signal power is concentrated in the quadrature component.

Given $f_{R, \Theta}(r, \theta)$, it is possible to find $f_{X, Y}(x, y)$ as $f_{X, Y}(x, y)=|J| f_{R, \Theta}(r, \theta)$, where $|J|=r$ is the Jacobian of the transformation $X=R \cos (\Theta)$ and $Y=R \sin (\Theta)$. Hence

$$
\begin{aligned}
f_{X, Y}(x, y) & \\
= & \frac{\alpha \mu^{\mu}\left(x^{2}+y^{2}\right)^{\mu(\alpha-2) / 2}|x|^{\mu(1+p)-1}|y|^{\mu(1-p)-1}}{2 \widehat{r}^{\alpha \mu} \Gamma(((1+p) / 2) \mu) \Gamma(((1-p) / 2) \mu)} \\
& \times \exp \left(-\frac{\mu}{\widehat{r}^{\alpha}}\left(x^{2}+y^{2}\right)^{\alpha / 2}\right)
\end{aligned}
$$

with $-\infty<x<\infty$ and $-\infty<y<\infty$. The expression in (6) is general and, to the best of the authors' knowledge, new. Notice that, in general, the in-phase and quadrature components are not independent random variables. As a consequence, the joint PDF of $X$ and $Y$ can not be factorized as a product of the marginal PDFs. It is found that the random variables $X$ and $Y$ are independent if and only if $\alpha=2$. In such a case

$$
\begin{aligned}
& \left.f_{X}(x)\right|_{\alpha=2} \\
& =\left(\frac{\mu}{\widehat{r}^{2}}\right)^{((1+p) / 2) \mu} \frac{|x|^{(1+p) \mu-1}}{\Gamma(((1+p) / 2) \mu)} \exp \left(-\frac{\mu x^{2}}{\widehat{r}^{2}}\right), \\
& \left.f_{Y}(y)\right|_{\alpha=2} \\
& =\left(\frac{\mu}{\widehat{r}^{2}}\right)^{((1-p) / 2) \mu} \frac{|y|^{(1-p) \mu-1}}{\Gamma(((1-p) / 2) \mu)} \exp \left(-\frac{\mu y^{2}}{\widehat{r}^{2}}\right) .
\end{aligned}
$$

Of course, $f_{X}(x)$ and $f_{Y}(y)$ given in (7) and (8) coincide, respectively, with those of (11) and (12) of [5].
The importance of (6) will become clear in Section 4 as it is used to compute the BER in a given subcarrier of the OFDM system. Another important aspect to be noticed about this joint PDF is that it does not provide the marginal PDFs of $X$ and $Y$ in a closed-form by integrating over $y$ or $x$, respectively. However, in the next section, we present an efficient method for generating the complex $\alpha-\mu$ without the knowledge of the marginal PDFs of $X$ and $Y$.

\section{Generating Complex $\alpha$ - $\mu$ Samples}

This section presents the method used to generate the complex $\alpha-\mu$ random variables for arbitrary values of its parameters. Bearing in mind that the nonlinearity parameter does not affect the phase statistics of the $\alpha$ - $\mu$ process, then its phase is given by that of the Nakagami- $m$ process. Therefore, in order to generate $\alpha-\mu$ complex variates, one can simply generate Nakagami- $m$ complex variates and use the relation between the envelopes of the two processes. Assuming that $Z_{N}$ and $Z$ are, respectively, Nakagami- $m$ and $\alpha-\mu$ complex variates, then, $R=|Z|=\left|Z_{N}\right|^{2 / \alpha}$ and $\Theta=\arg (Z)=\arg \left(Z_{N}\right)$. Now, from (7) and (8), it can be seen that the in-phase and quadrature components of the Nakagami- $m$ have a Gamma PDF with symmetry around zero. Hence, the following can be written:

$$
Z_{N}=S_{X} \sqrt{\frac{G_{X}}{\mu}}+j S_{Y} \sqrt{\frac{G_{Y}}{\mu}},
$$

where $G_{X} \sim \operatorname{Gamma}\left((1+p) \mu / 2, \widehat{r}^{2}\right)$ and $G_{Y} \sim \operatorname{Gamma}((1-$ p) $\left.\mu / 2, \widehat{r}^{2}\right)$ are independent Gamma random variables, $\mu$ accounts for the fading parameter, and $\hat{r}^{2}=\mathbb{E}\left[\left|Z_{N}\right|^{2}\right]$. The multiplicative factors $S_{X}$ and $S_{Y}$ are independent discrete random variables assuming the values 1 and -1 with equal probabilities. Any mathematical software tool (e.g., Mathematica) that provides Gamma variate generator can be used here. Then to create the complex $\alpha-\mu$ random variable, it suffices to make the transformation

$$
Z=\left|Z_{N}\right|^{2 / \alpha} \exp \left(j \arg \left(Z_{N}\right)\right) .
$$

It should be mentioned that the fact that the marginal PDFs for the in-phase and quadrature components of the $\alpha-\mu$ process have not been obtained in closed-form did not affect the generation of the $\alpha-\mu$ complex samples. The PDFs closedform issue has been circumvented by generating complex samples for Nakagami- $m$ variates, whose marginal PDFs are given in closed-form, and then applying the appropriate transformation of variables as in (10), from which the $\alpha-\mu$ samples result.

When compared to other generation methods of complex Nakagami- $m$ variates, such as the Acceptance-Rejection method used in [22], the proposed method here has a dramatically better efficiency.

In order to validate this new method, we show some plots comparing theoretical (solid lines) and simulated (symbols) curves. Each curve was generated using $5 \times 10^{5}$ samples with $\mu=3$, where, without loss of generality, $\widehat{r}^{\alpha}=1$ was used. Figure 1 depicts various shapes of the $\alpha-\mu$ envelope density. 


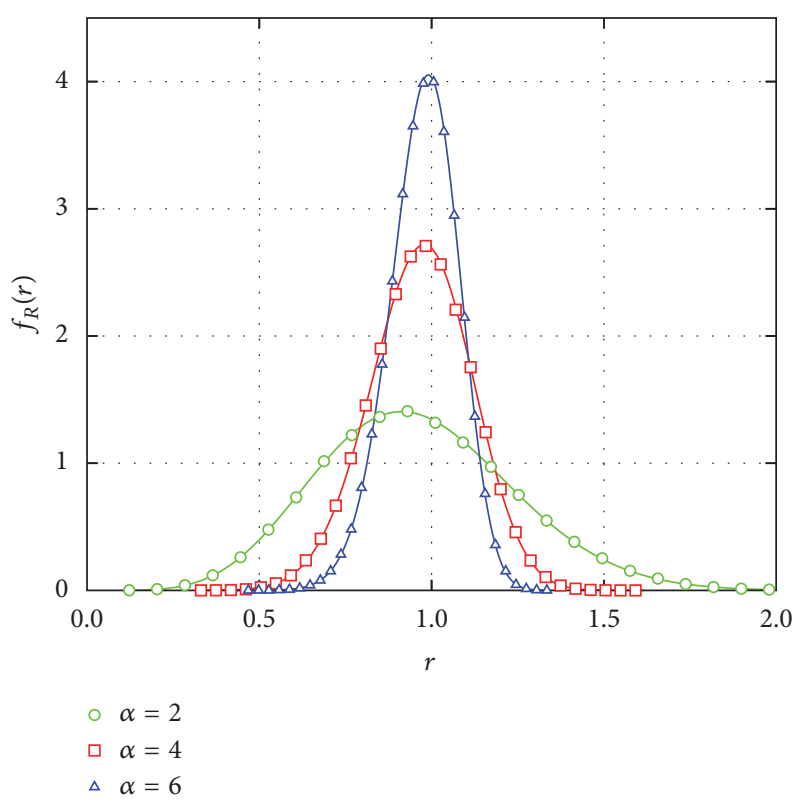

FIgURE 1: Empirical and theoretical $\alpha-\mu$ envelope distribution with $\mu=3$ and irrelevant $p$.

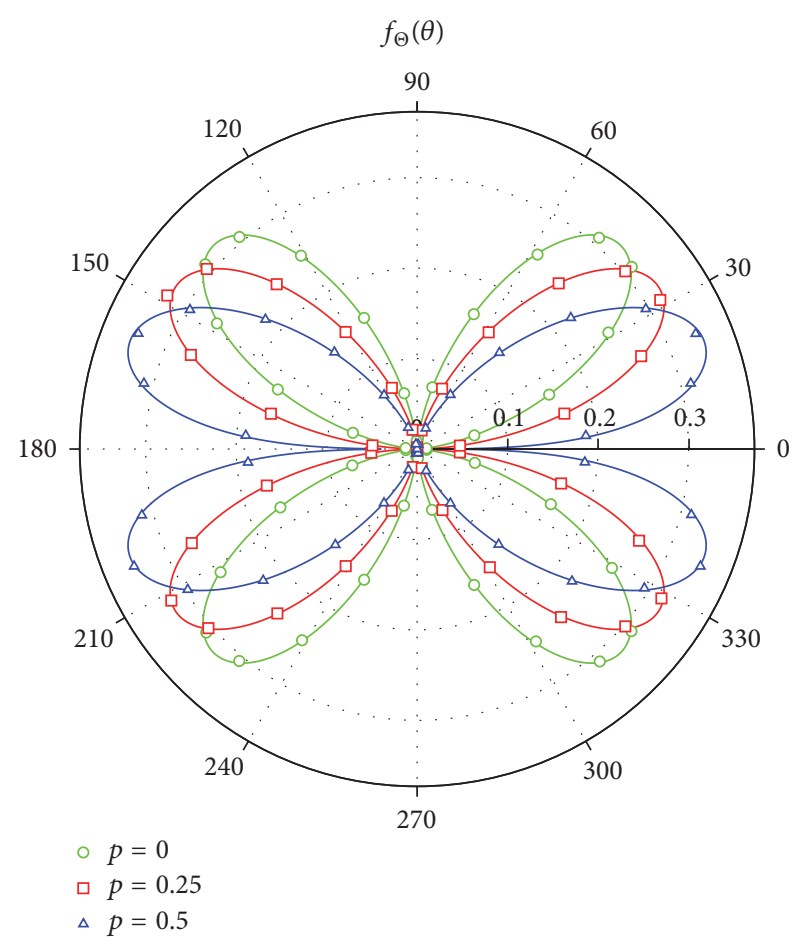

FIGURE 2: Empirical and theoretical $\alpha-\mu$ phase distribution with $\mu=$ 3 and irrelevant $\alpha$.

Figure 2 shows the corresponding shapes of the $\alpha-\mu$ phase PDF, plotted in polar coordinates.

In Figures 3 and 4, the PDFs of real and imaginary parts of the $\alpha-\mu$ distribution are plotted, respectively. In all plots, we consider the parameter $\mu=3$, and we vary the parameters $\alpha$ and $p$ to verify the correctness of the proposed method to generate the $\alpha-\mu$ complex density. We can notice the excellent

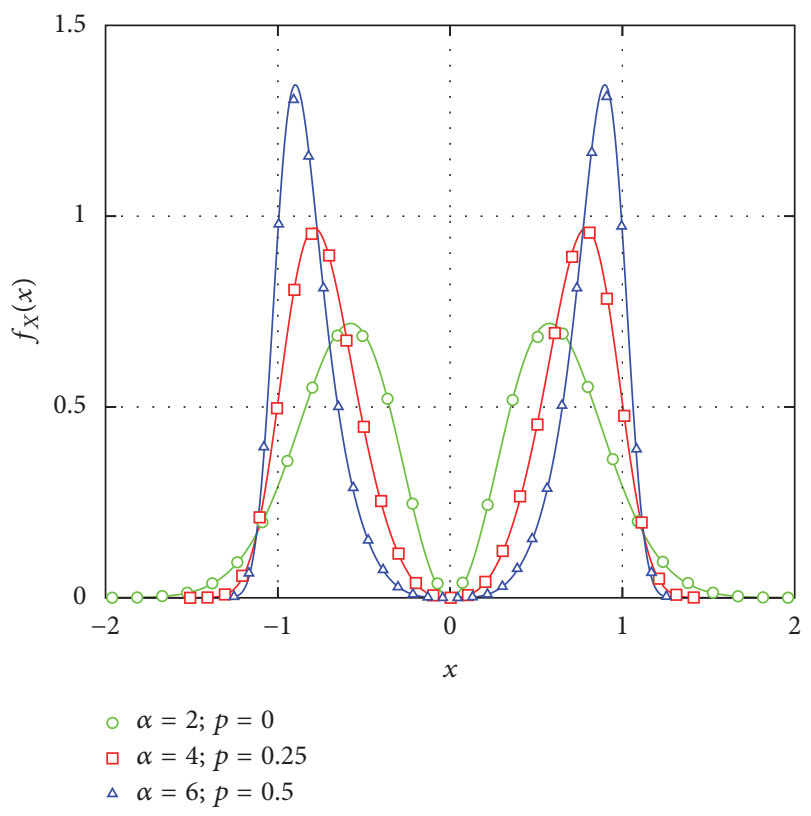

FIGURE 3: Empirical and theoretical $f_{X}(x)$ distribution with $\mu=3$.

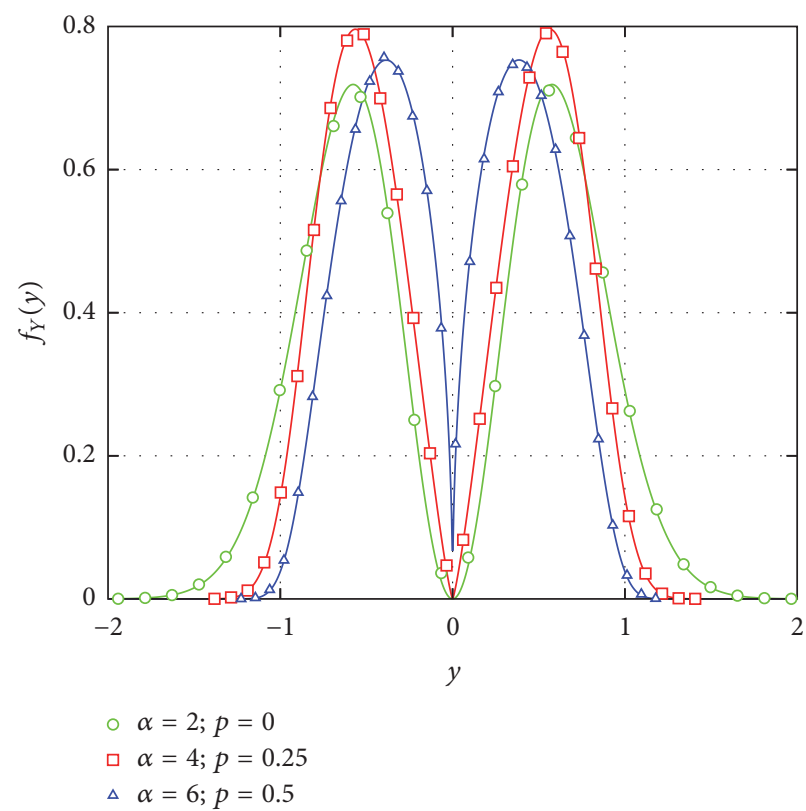

FIGURE 4: Empirical and theoretical $f_{Y}(y)$ distribution with $\mu=3$.

agreement between the simulation and the theoretical curves. It is worth mentioning that the theoretical curves of real and imaginary parts were calculated numerically, because they do not lend themselves into closed-form expressions.

\section{OFDM System under the Complex $\alpha-\mu$ Channel}

4.1. Theoretical Expression for the BER. The OFDM system under investigation here is an idealized one, in which issues like channel estimation by training, cyclic prefix length, 
equalization strategy, the presence of narrowband interference, impulse noise, and transceiver impairments, and others are overlooked. To accomplish the analysis of an OFDM system under the complex $\alpha-\mu$ channel presented in this paper, a framework developed in [20] is used. In a simplified manner, the BER is computed combining three equations [20]:

(1) The Characteristic Function:

$$
\begin{gathered}
\Phi_{\ell}(u, v)=\mathbb{E}\left[e^{-j X_{\ell}\{u \cos (2 \pi(n / N) \ell)+v \sin (2 \pi(n / N) \ell)\}}\right. \\
\left.\times e^{-j Y_{\ell}\{u \sin (2 \pi(n / N) \ell)-v \cos (2 \pi(n / N) \ell)\}}\right]
\end{gathered}
$$

where (i) the subscript $\ell$ denotes $\ell$ th path with $\ell=$ $0,1,2, \ldots, L-1$ in which $L$ is the total number of multipath rays; (ii) $X_{\ell}$ and $Y_{\ell}$ are the real and imaginary part of the complex $\alpha-\mu$ channel at the $\ell$ th path, respectively; (iii) $N$ is the total number of subcarriers with $n=0,1,2, \ldots, N-1$ being the index of the subcarrier.

(2) The Moment Generating Function:

$$
\begin{aligned}
\mathscr{M}(z)= & \mathbb{E}\left[e^{-z\left|H_{n}\right|^{2}}\right] \\
= & \frac{1}{\pi} \int_{-\infty}^{\infty} \int_{-\infty}^{\infty} e^{-\left(u^{2}+v^{2}\right)} \\
& \times \prod_{\ell=0}^{L-1} \Phi_{\ell}(2 \sqrt{z} u, 2 \sqrt{z} v) d u d v,
\end{aligned}
$$

where $H_{n}=\sum_{\ell=0}^{L-1} Z_{\ell} e^{-j 2 \pi n \ell / N}$ is the frequencydomain channel impulse response and $Z_{\ell}, \ell=0,1$, $2, \ldots, L-1$, are independent complex $\alpha-\mu$ random variables given by (1) with the appropriate subscript.

(3) The BER of BPSK ((13) is slightly different from that of [20, eqn. (24)] due to typo found there):

$$
P_{b}=\frac{1}{\pi} \int_{0}^{\pi / 2} \mathscr{M}^{D}\left(\frac{\mathrm{SNR}}{2 \sin ^{2}(\theta)}\right) d \theta,
$$

where $D$ is the order of diversity of the maximum ratio combiner (MRC) and SNR is the signal-to-noise ratio.

For the $\alpha-\mu$ channel, however, (11) has to be computed numerically as

$$
\begin{aligned}
& \Phi_{\ell}(u, v) \\
& \quad=\int_{-\infty}^{\infty} \int_{-\infty}^{\infty} f_{X_{\ell}, Y_{\ell}}\left(x_{\ell}, y_{\ell}\right) e^{-j\left(x_{\ell} w_{x_{\ell}}+y_{\ell} w_{y_{\ell}}\right)} d x_{\ell} d y_{\ell},
\end{aligned}
$$

where $f_{X_{\ell}, Y_{\ell}}\left(x_{\ell}, y_{\ell}\right)$ is the joint PDF of the in-phase and quadrature components of the channel at the $\ell$ th path given by (6), $w_{x_{\rho}}=\{u \cos (2 \pi(n / N) \ell)+v \sin (2 \pi(n / N) \ell)\}$, and $w_{y_{\ell}}=$ $\{u \sin (2 \pi(n / N) \ell)-v \cos (2 \pi(n / N) \ell)\}$.

It is worth mentioning that (13) can be easily generalized to other modulations schemes such as the $M$-PSK $[1$, eq. (5.67)] and $M$-QAM modulations [1, eq. (8.110)].

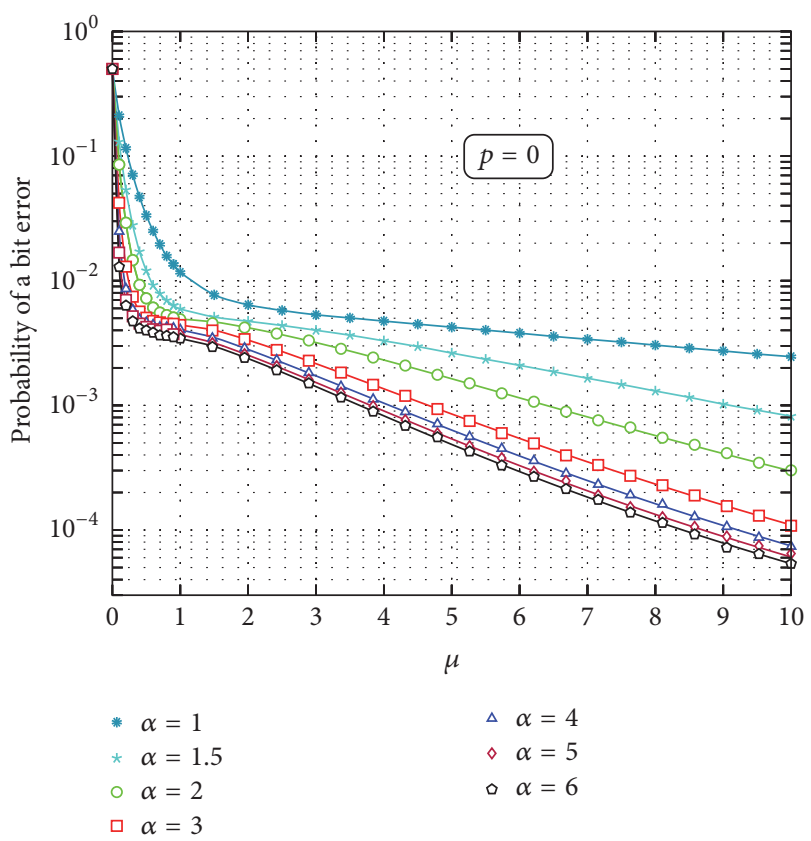

FIgURE 5: Average BER against the fading parameter $\mu$ under $\alpha-\mu$ fading channel with SNR $=20 \mathrm{~dB}, L=3$, and $p=0$.

4.2. Numerical Examples. This section assesses the performance of the BPSK-based OFDM system in terms of BER concerning the influence of the main system parameters. All experiments depicted here consider $n=N / 2$, as also used in [20], and $\sum_{\ell=0}^{L-1} \mathbb{E}\left[R_{\ell}^{2}\right]=1$. The theoretical results are calculated numerically using (13). Independent simulations have been performed so as to validate the theoretical expressions. MATLAB was used to implement the code according to the models described throughout the paper. For each point we generate at least $2 \times 10^{7}$ samples using the Monte Carlo approach.

In Figure 5, the theoretical probability error of a bit (solid lines) and the estimated through simulation bit error rate (symbols) are plotted against the parameter $\mu$. The nonlinearity parameter $\alpha$ varies as $\{1 ; 1.5 ; 2 ; 3 ; 4 ; 5 ; 6\}$ and $L=3$ for a fixed value of $p=0$ (balanced scenario). Note the excellent agreement between theoretical and simulated curves showing the usefulness and correctness of the analytical procedure. It is worth mentioning that for $\alpha=2$, that is, the Nakagami$m$ case, the result is the same as the one obtained in [20], as expected. It is possible to conclude that the increase of the clustering parameter $\mu$ diminishes the BER for a fixed value of $\alpha$. This is an expected result, since the parameter $\mu$ accounts for the number of multipath clusters. In other words, the higher the value of $\mu$ is, the less severe the fading is. Hence the performance in terms of probability of error is improved. In the same way, the increase of the parameter $\alpha$ also improves the BER performance. Of course, the higher the values of $\alpha$ are, the more deterministic the channel is and, therefore, the less vulnerable to variation the channel is.

In order to assess the impact of the phase parameter, Figure 6 depicts the same set of curves of Figure 5 but now with $p=1 / 3$, corresponding to a case in which the power 


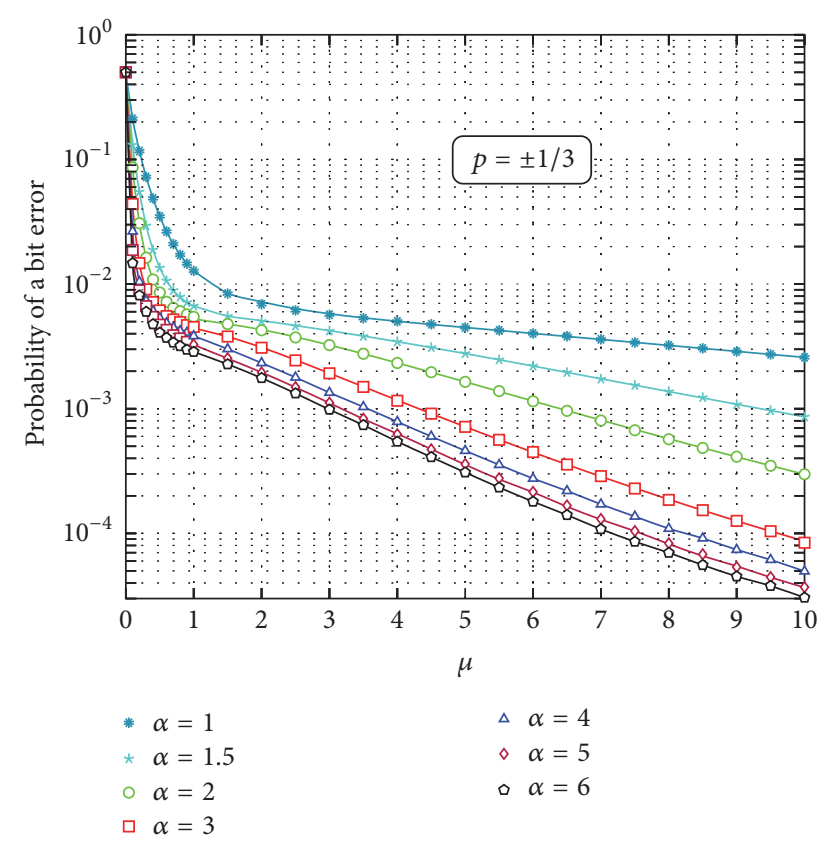

FIGURE 6: Average BER against the fading parameter $\mu$ under $\alpha-\mu$ fading channel with SNR $=20 \mathrm{~dB}, L=3$, and $p=1 / 3$.

(or the number of clusters) of the in-phase component is the double as that of the quadrature component. In addition to the conclusions already drawn from Figure 5, by comparing the curves for the same nonlinearity parameter $\alpha$, for $\alpha>2$, the increase in the phase parameter leads to a lower BER. Furthermore, the performance obtained for $p=0$ (balanced) and $p=1 / 3$ (imbalanced) scenarios improves as $\alpha$ increases. However, for $\alpha \leq 2$ the performance under the variation of the phase parameter $p$ is practically the same for any value of $\alpha$. This means that the power imbalance between inphase and quadrature components has very little effect on the system performance in terms of BER. Moreover, the impact of phase imbalance on the system performance is greater for high values of $\alpha$.

With the objective of clarifying even more the impact of phase imbalance, Figure 7 depicts the same set of curves of Figure 5 but now against the absolute value of the parameter $p$ for fixed $\mu=3$. Firstly, all the previous conclusions are corroborated by this new graph. Additionally, for $\alpha \leq 2$ the impact of phase imbalance on the system performance is greater for high values of $|p|$ increasing slightly the BER. In contrast, for $\alpha>2$, for high value of the power imbalance between in-phase and quadrature components, the effect on the system performance in terms of BER is higher. It is desirable to have an intuitive explanation for such a behavior. Unfortunately, the problem is rather intricate and nonlinear, so further investigation is due.

In Figure 8, the BER is plotted against the SNR for fixed $\mu=3$ and $L=3$ using the balanced $(p=0)$ and imbalanced $(p=1 / 3)$ scenarios for single $(D=1)$ and dual $(D=2)$ channel diversity reception. In this figure the influence of $\alpha$ and the diversity parameter $D$ is clearly noticed. As expected,

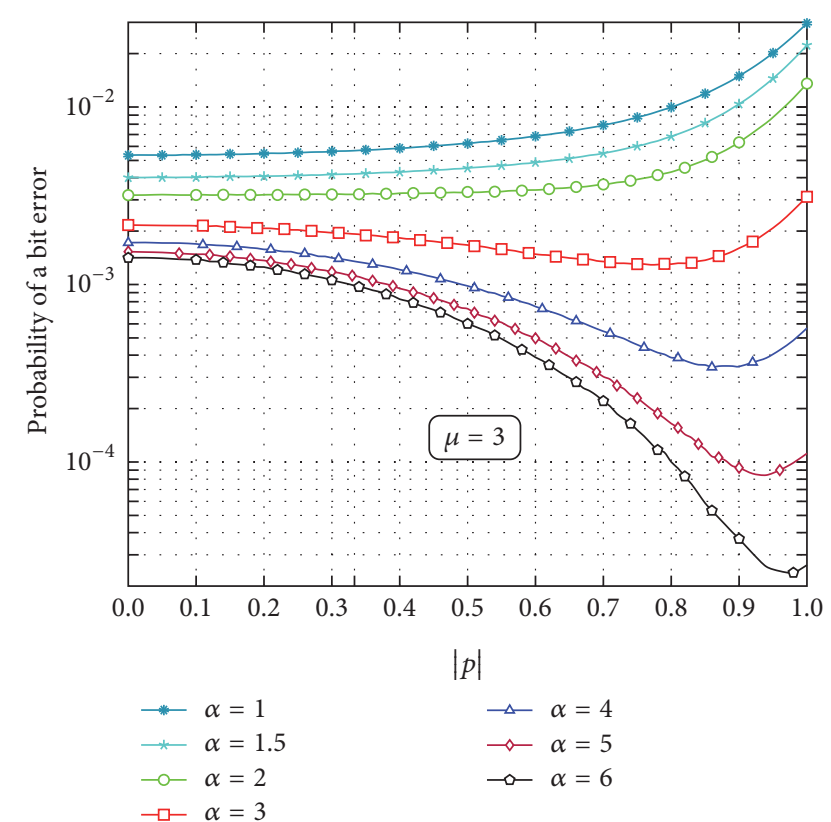

Figure 7: Average BER against the phase parameter $p$ under $\alpha-\mu$ fading channel with SNR $=20 \mathrm{~dB}, L=3$, and $\mu=3$.

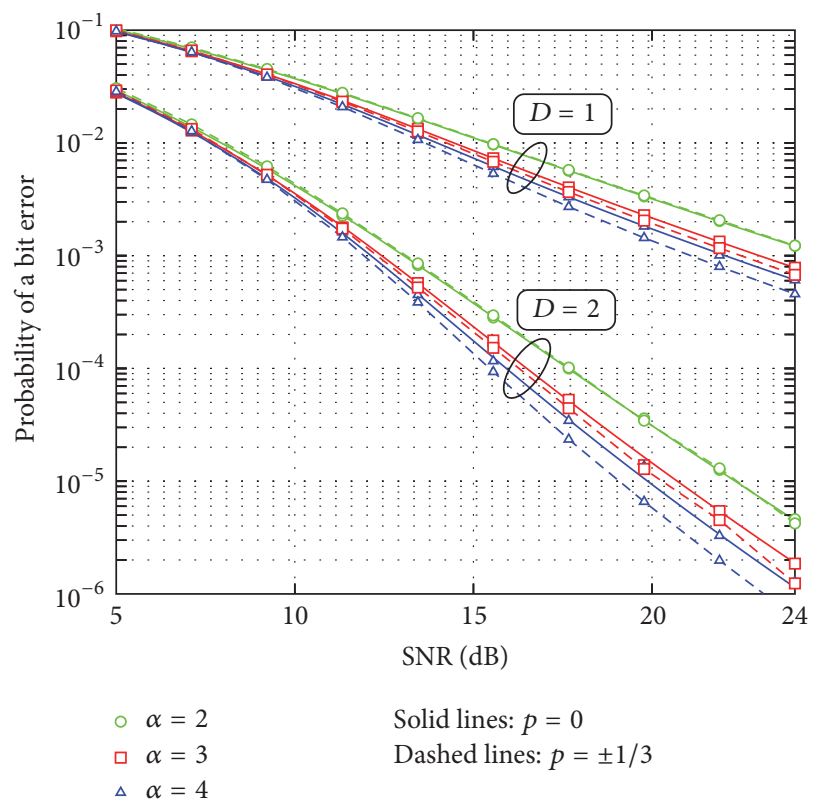

FIgURE 8: Average BER against SNR for single and dual channel diversity reception with $\mu=3$ and $L=3$ for balanced and unbalanced scenarios.

for higher diversity system order, the performance in terms of probability of error is improved. The difference between the BER of the single and dual channel diversity reception increases at higher SNR values. Again, phase imbalance impacts positively on the system performance, especially for high values of SNR. 


\section{Conclusions}

In this paper, a model for the complex $\alpha-\mu$ channel was presented. From such a model, the joint probability density function of in-phase and quadrature components was found. This was then used within an analytical framework to assess the bit error rate performance of an OFDM system. As a byproduct of the main results, an efficient, simple, and general complex $\alpha-\mu$ variates generator was proposed. It was found that an increase of the value of the nonlinearity parameter as well as of the number of clusters improves the system performance in terms of its bit error rate. In the same way, the imbalance of power (or clustering) between in-phase and quadrature components also has a positive impact on such performance.

\section{Conflicts of Interest}

The authors declare that there are no conflicts of interest regarding the publication of this paper.

\section{Acknowledgments}

This work was partially supported by Finep, with resources from Funttel, Grant no. 01.14.0231.00, under the Radiocommunication Reference Center (Centro de Referência em Radiocomunicações, CRR) project of the National Institute of Telecommunications (Instituto Nacional de Telecomunicações, Inatel), Brazil.

\section{References}

[1] M. K. Simon and M. Alouini, Digital Communication Over Fading Channels, John Wiley \& Sons, Inc., New York, USA, 2000.

[2] P. M. Shankar, "Statistical models for fading and shadowed fading channels in wireless systems: A pedagogical perspective," Wireless Personal Communications, vol. 60, no. 2, pp. 191-213, 2011.

[3] M. D. Yacoub, “The $\alpha-\mu$ distribution: a physical fading model for the Stacy distribution," IEEE Transactions on Vehicular Technology, vol. 56, no. 1, pp. 27-34, 2007.

[4] M. D. Yacoub, G. Fraidenraich, and J. C. S. Santos Filho, "Nakagami-m phase-envelope joint distribution," Electronics Letters, vol. 41, no. 5, pp. 259-261, 2005.

[5] M. D. Yacoub, "Nakagami-m phase-envelope joint distribution: A new model," IEEE Transactions on Vehicular Technology, vol. 59, no. 3, pp. 1552-1557, 2010.

[6] I. B. G. Porto, M. D. Yacoub, J. C. S. Santos Filho, S. L. Cotton, and W. G. Scanlon, "Nakagami-m phase model: Further results and validation," IEEE Wireless Communications Letters, vol. 2, no. 5, pp. 523-526, 2013.

[7] L. Rayleigh, "On the resultant of a large number of vibrations of same pitch and of arbitrary phase," Philosophical Magazine, vol. 10 , no. 60 , pp. $73-78,1880$.

[8] R. S. Hoyt, "Probability functions for the modulus and angle of the normal complex variate," The Bell System Technical Journal, vol. 26, pp. 318-359, 1947.
[9] S. O. Rice, "Statistical Properties of Random Noise Currents," in Statistical Properties of Random Noise Currents, N. Wax, Ed., NY, USA: Dover, 1954.

[10] Statistical Methods in Radio Wave Propagation, Elsevier, 1960.

[11] W. Weibull, "A statistical distribution function of wide applicability," Journal of Applied Mechanics, vol. 18, pp. 293-297, 1951.

[12] J. Reig, M.-T. Martínez-Inglés, L. Rubio, V.-M. RodrigoPeñarrocha, and J. Molina-García-Pardo, "Fading evaluation in the $60 \mathrm{GHz}$ band in line-of-sight conditions," International Journal of Antennas and Propagation, vol. 2014, Article ID 984102, 12 pages, 2014.

[13] M. D. Yacoub, “The $\alpha-\eta-\kappa-\mu$ fading model," IEEE Transactions on Antennas and Propagation, vol. 64, no. 8, pp. 3597-3610, 2016.

[14] G. Berardinelli, K. I. Pedersen, T. B. Sørensen, and P. Mogensen, "Generalized DFT-Spread-OFDM as 5G Waveform," IEEE Communications Magazine, vol. 54, no. 11, pp. 99-105, 2016.

[15] G. Berardinelli, F. M. L. Tavares, T. B. Sørensen, P. Mogensen, and K. Pajukoski, "Zero-tail DFT-spread-OFDM signals," in Proceedings of the 2013 IEEE Globecom Workshops, GC Wkshps 2013, pp. 229-234, usa, December 2013.

[16] A. Sahin, R. Yang, M. Ghosh, and R. L. Olesen, "An improved unique word DFT-spread OFDM scheme for 5G systems," in Proceedings of the IEEE Globecom Workshops, GC Wkshps 2015, USA, December 2015.

[17] N. Michailow, M. Matthe, I. S. Gaspar et al., "Generalized frequency division multiplexing for 5 th generation cellular networks," IEEE Transactions on Communications, vol. 62, no. 9, pp. 3045-3061, 2014.

[18] B. Farhang-Boroujeny and H. Moradi, "OFDM Inspired Waveforms for 5G," IEEE Communications Surveys and Tutorials, vol. 18, no. 4, pp. 2474-2492, 2016.

[19] X. Zhang, L. Chen, J. Qiu, and J. Abdoli, “On the Waveform for 5G," IEEE Communications Magazine, vol. 54, no. 11, pp. 74-80, 2016.

[20] K. A. Hamdi, "Analysis of OFDM over Nakagami-m fading with nonuniform phase distributions," IEEE Transactions on Wireless Communications, vol. 11, no. 2, pp. 488-492, 2012.

[21] M. Abramowitz and I. A. Stegun, Handbook of Mathematical Functions, 1965.

[22] R. A. A. de Souza, R. Cogliatti, and M. D. Yacoub, "Efficient acceptance-rejection method for Nakagami-m complex samples," IEEE Wireless Communications Letters, vol. 3, no. 1, pp. 94-96, 2014. 


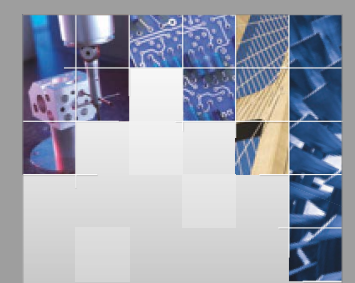

\section{Enfincering}
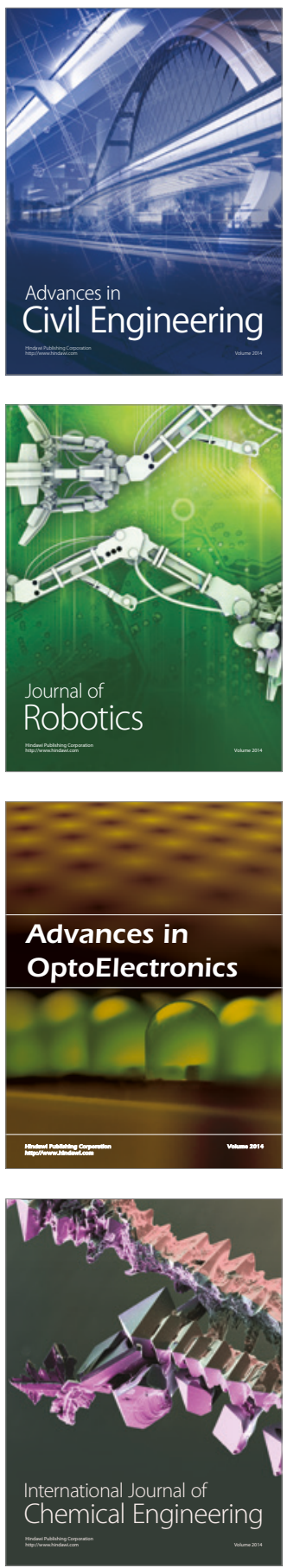

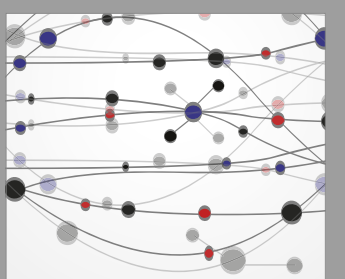

The Scientific World Journal

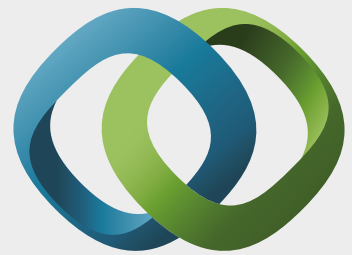

\section{Hindawi}

Submit your manuscripts at

https://www.hindawi.com
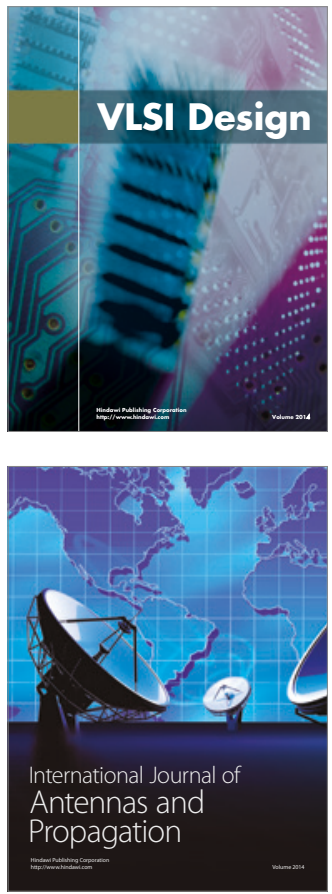

\section{Rotating}

Machinery
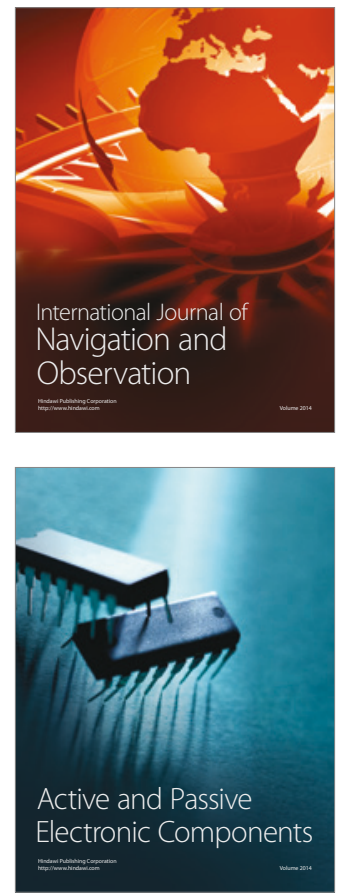
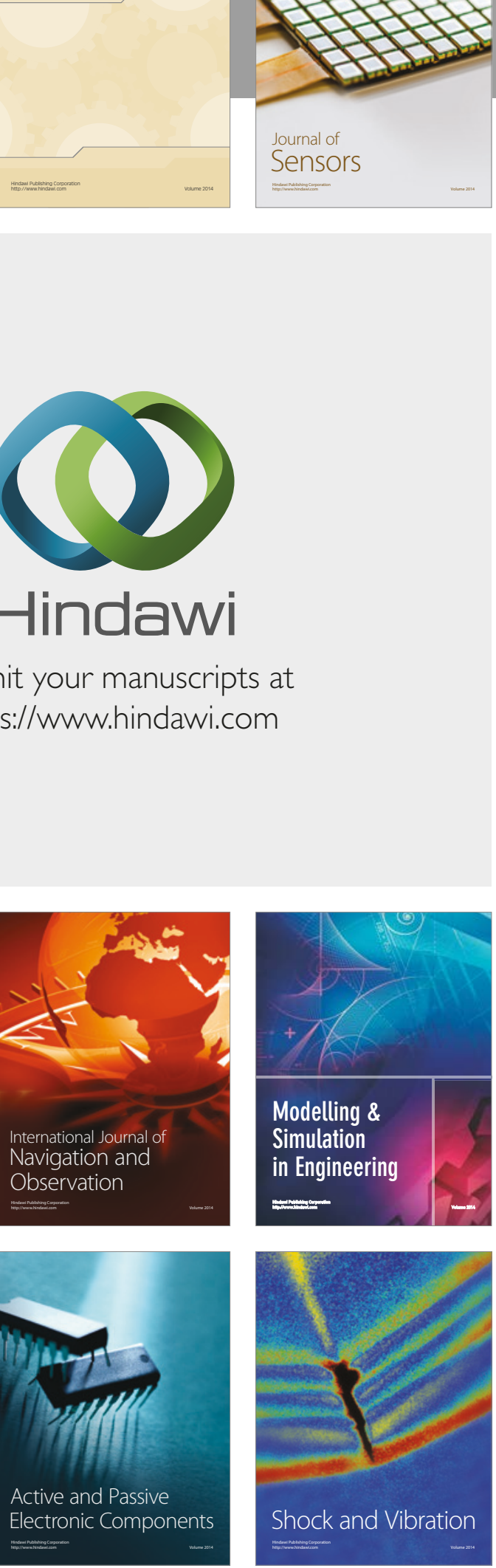
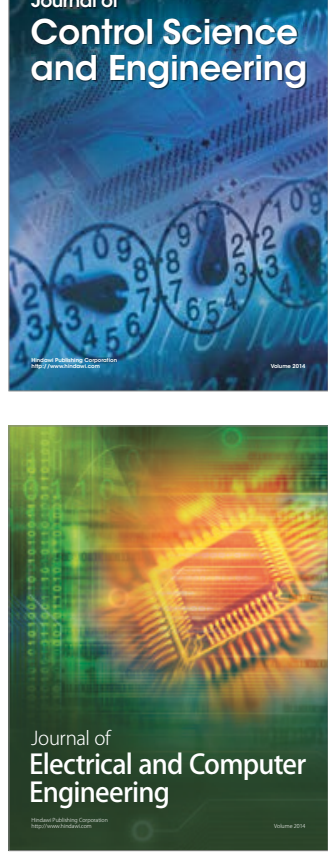

Distributed

Journal of

Control Science

and Engineering
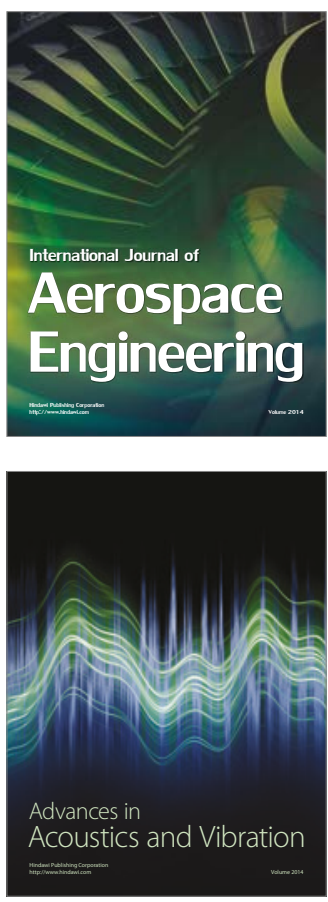

Sensor Networks 